\title{
CONJUGATES OF DIFFERENTIABLE FLOWS
}

GORDON G. JOHNSON

(Received 4 November 1970; revised 14 April 1971)

Communicated by B'Navd

The work in this paper is directed at the question: What differentiable flows on $[0,1][1]$ are conjugates of linear fractional flows on $[0,1]$ ?

Lemma. If $h$ is a homeomorphism of $[0,1]$ onto $[0,1]$ such that for some number $a \in(0,1] h$ has a continuous positive derivative on $(0, a]$ and there is a number $r$ such that $\lim _{x \rightarrow 0} x^{r} h^{\prime}(x)>0$ then $r<1$.

Proof. Suppose $r \geqq 1$ and $\lim _{x \rightarrow 0} x^{r} h^{\prime}(x)=A>0$. Then there is a number $b \in(0, a]$ such that if $x \in(0, b]$ then $x^{r} h^{\prime}(x)>A / 2$. Since $r \geqq 1$ we have that $x^{r} h^{\prime}(x) \leqq\left(x^{r} h(x)\right)^{\prime}$ and hence that

$$
\int_{0}^{b}\left(x^{r} h(x)\right)^{\prime} d x \geqq \int_{0}^{b} x^{r} h^{\prime}(x) d x \geqq A b / 2 .
$$

It then follows that $b^{r} h(b) \geqq A b / 2$ and hence that $d^{r-1} h(d) \geqq A / 2$ if $d \in(0, b]$. This is impossible, hence $r<1$.

Definition. A differentiable flow $F_{t}$ on $[0,1]$ is said to be of type $I$ if

1. 0 and 1 are the only fixed points of $F_{t}$,

2. $F_{t}^{\prime}(0)=c^{t}, F_{t}^{\prime}(1)=d^{t}$ where $c>1>d$ and

3. there are homeomorphisms $\phi$ and $\Phi$ from $[0,1)$ and $(0,1]$ respectively onto $[0, \infty)$ each having a continuous nonzero derivative such that $F_{\imath}=\phi^{-1}\left(c^{t} \phi\right)$ $=\Phi^{-1}\left(d^{t} \Phi\right)$.

TheOREM. A necessary and sufficient condition that a differentiable flow $F_{t}$ on $[0,1]$ be of type $I$ is that there is:

1. a homeomorphism $h$ of $[0,1]$ onto $[0,1]$ which has a continuous positive derivative on $(0,1]$ and a number $r$ such that $\lim _{x \rightarrow 0} x^{r} h^{\prime}(x)>0$. and

2. a linear fractional flow $L_{t}$ on $[0,1]$ such that $F_{t}=h \circ L_{t} \circ h^{-1}$.

Proof. Suppose $F_{t}$ is a differentiable flow of type I with $F_{t}^{\prime}(0)=c^{t}, F^{t}(1)=d^{t}$ where $c>1>d$.

Define a linear fractional flow $L_{t}$ by 


$$
L_{t}(x)=d^{-t} x /\left[\left(d^{-t}-1\right) x+1\right] \text { if } x \in[0,1] \text { and } t \in(-\infty, \infty) .
$$

Fix $b \in(0,1)$ and define a function $h^{-1}$ by

$$
\begin{gathered}
h^{-1}(x)=\left[1+y(\phi(x))^{-p}\right]^{-1} \text { if } 0 \leqq x<1 \text { and } \\
h^{-1}(1)=1
\end{gathered}
$$

where $p=-\ln d / \ln c$ and $y=(1-b) / b(\phi(b))^{p}$.

It is easily verified that $h^{-1}$ is a homeomorphism of $[0,1]$ onto $[0,1]$ which has a positive continuous derivative on $(0,1)$. Also a sequence of straightforward computations establishes that $F_{t}=h \circ L_{t} \circ h^{-1}$.

Because $h^{\prime} \circ h^{-1}(x)=\left[(\phi(x))^{p}+y\right]^{2} / y p(\phi(x))^{p-1} \phi^{\prime}(x)$ we have that

$$
\left(h^{-1}(x)\right)^{r} h^{\prime} \circ h^{-1}(x)=[\phi(x)+y]^{2-r} / y p \phi^{\prime}(x)
$$

where $r=1-1 / p$. It then follows that

$$
\lim _{x \rightarrow 0} x^{r} h^{\prime}(x)=y^{1-r} / p \phi^{\prime}(0)>0 .
$$

All that remains to establish the result going one way, is that $h$ has a continuous positive derivative on $(0,1]$. This follows from the following observations. Fix $k \in(0,1)$ and recall that

$$
\phi^{-1}\left(c^{t} \phi(k)\right)=\Phi^{-1}\left(d^{t} \Phi(k)\right) .
$$

If $m=\phi(k), \quad n=\Phi(k)$ and $z=\Phi^{-1}\left(d^{t} n\right)$ then $\Phi(z)=n d^{t}$ and hence $t=\ln \left[\Phi\left(\frac{1}{2}\right) / n\right] / \ln d$. We then have that

$$
\phi(z)=m c^{\ln [\Phi(z) / n] / \ln d}=m(\Phi(z) / n)^{\ln c / \ln d} .
$$

Therefore $(\phi(z))^{p}=m^{\prime} n / \Phi(z)$ and $h^{-1}(z)=\left[1+y \Phi(z) / m^{\prime} n\right]^{-1}$, where $m^{\prime}=m^{p}$. Hence $h^{-1}$ has a continuous positive derivative on $(0,1]$.

To establish the remaining half of the theorem suppose $h$ is a homeomorphism of $[0,1]$ onto $[0,1]$ having the required properties, and that $L_{t}$ is a linear fractional flow on $[0,1]$ with $L_{t}^{\prime}(0)=a^{t}$ where $a>1$. Let $F_{t}=h \circ L_{t} \circ h^{-1}$. Clearly $F_{t}$ is a flow on $[0,1]$ which is a differentiable flow on $(0,1]$, also only 0 and 1 are fixed points on $F_{t}$.

Since $F_{t}^{\prime}(x)=h^{\prime} \circ L_{t} \circ h^{-1}(x) \cdot L_{t}^{\prime} \circ h^{-1}(x) \cdot h^{-1}(x)$ we have that

$$
\begin{aligned}
\lim _{x \rightarrow 0} F_{t}^{\prime}(x) & =\lim _{x \rightarrow 0} h^{\prime} \circ L_{t}(x) \cdot L_{t}^{\prime}(x) / h^{\prime}(x) \\
& =\lim _{x \rightarrow 0}\left(L_{t}(x)\right)^{r} h^{\prime} \circ L_{t}(x) \cdot x^{r} L_{t}^{\prime}(x) /\left(L_{t}(x)\right)^{r} x^{r} h^{\prime}(x) \\
& =\left(L_{t}^{\prime}(0)\right)^{1-r} \\
& =a^{(1-r) t} .
\end{aligned}
$$


Note that $F_{t}^{\prime}(1)=L_{t}^{\prime}(1)=a^{-t}$, thus $a^{1-r}>1>a^{-1}$.

We need only to produce the desired homeomorphisms $\phi$ and $\Phi$ to complete the argument.

Define a function $\theta$ on $(0,1]$ by

$$
\theta(x)=h^{\prime}(1) /\left[h^{\prime} \circ h^{-1}(x)\left(h^{-1}(x)\right)^{2}\right] \text { for } x \text { in }(0,1] .
$$

It is clear that $\theta$ is continuous and positive on $(0,1]$.

If $\Phi(x)=\int_{x}^{1} \theta$ then there is a number $B>0$ such that $\Phi(x)=B\left[\left(1 / h^{-1}(x)\right)-1\right]$ and hence $\Phi$ is a homeomorphism of $(0,1]$ onto $[0, \infty)$ which has a negative continuous derivative on $(0,1]$. Moreover, a sequence of computations yields

$$
\begin{aligned}
\Phi^{-1}\left(a^{-t} \Phi(x)\right) & =h\left[B /\left(a^{-t} \Phi(x)+B\right)\right] \\
& =h \circ L_{t} \circ h^{-1}(x) \\
& =F_{t}(x) .
\end{aligned}
$$

Now define a function $\phi$ on $[0,1)$ by

and

$$
\phi(x)=(\Phi(x))^{r-1} \text { if } x \in(0,1)
$$

$$
\phi(0)=0 \text {. }
$$

Hence $\phi$ is a homeomorphism of $[0,1)$ onto $[0, \infty)$ which has a positive continuous derivative on $(0,1)$.

Now $\quad \phi^{\prime}(x)=(r-1)(\Phi(x))^{r-2} \Phi^{\prime}(x)$

$$
=(1-r) B^{r-2} \theta(x)\left[\left(1 / h^{-1}(x)\right)-1\right]^{r-2} \text { on }(0,1) \text {. }
$$

Using the definitions of $\theta$ and $h^{-1}$ and the above we have that

$$
\begin{aligned}
\phi^{\prime}(x) & =\left[(1-r) B^{r-2} h^{\prime}(1) / h^{\prime} \circ h^{-1}(x)\left(h^{-1}(x)\right)^{2}\right]\left[\left(1 / h^{-1}(x)\right)-1\right]^{r-2} \\
& =A\left[1-h^{-1}(x)\right]^{r-2} /\left(h^{-1}(x)\right)^{r} h^{\prime} \circ h^{-1}(x)
\end{aligned}
$$

where $A=(1-r) B^{r-2} h^{\prime}(1)$.

Hence $\lim _{x \rightarrow 0} \phi^{\prime}(x)>0$ and therefore $\phi$ has a positive continuous derivative on $[0,1)$. Also a simple computation shows that

$$
F_{1}=\phi^{-1}\left(a^{(1-r) t} \phi\right)
$$

which concludes the proof of the theorem.

\section{References}

[1] M. K. Fort Jr., 'The embedding of homeomorphisms in flows', Proc. Amer. Math. Soc. 6( 1955), 960-967. MR18, 326. 
[2] N. J. Fine and F. F. Schweigert, 'On the group of homeomorphisms of an arc', Ann. of Math. (2) 62 (1955), 237-253, MR17, 288.

[3] N. E. Foland and W. R. Utz, 'The embedding of discrete flows in continuous flows,' Proc. Internat. Sympos. Ergodic Theory (Tulane Univer., New Orleans, La., 1961, Academic Press, New York, 1963, pp. 121-139), MR 28 \# 3412.

[4] P. F. Lam, The problem of embedding a homeomorphism in a flow subject to differentiability conditions (Ph. D. Thesis, Yale University 1967).

Virginia Polytechnic Institute \& State University

Blacksburg, Virginia 24601

U.S.A.

Present address.

University of Houston,

Houston, Texas 77004

U. S. A. 